\title{
Is there a role for telemedicine in adult allergy services?
}

\author{
Mamidipudi T. Krishna+\#, Rebecca C. Knibb\# and Aarnoud P. Huissoon+ \\ +Heart of England NHS Foundation Trust (HEFT), Birmingham, UK \\ \#Aston University, Birmingham, UK
}

Short title: Telemedicine and adult allergy service

\section{$\underline{\text { Address for correspondence }}$}

Dr. M. T. Krishna

Department of Allergy and Immunology

Birmingham Heartlands Hospital

Bordesley Green East

Birmingham B9 5SS

Email:mtkrishna@yahoo.com

Tel: +441214241807

Fax: +44 1214243229 


\section{Abstract}

Telemedicine refers to the application of telecommunication and information technology (IT) in the delivery of health and clinical care at a distance or remotely and can be broadly considered in two modalities: store-and-forward and real-time interactive services.

Preliminary studies have shown promising results in radiology, dermatology, intensive care, diabetes, rheumatology and primary care. However, the evidence is limited and hampered by small sample sizes, paucity of randomised controlled studies and lack of data relating to costeffectiveness, health related quality of life and patient and clinician satisfaction. This review appraises the evidence from studies that have employed telemedicine tools in other disciplines and makes suggestions for its potential applications in specific clinical scenarios in adult allergy services. Possible examples include: triaging patients to determine the need for allergy tests; pre-assessment for specialised treatments such as allergen immunotherapy; follow up to assess treatment response and side effects; and education in self-management plan including training updates for self-injectable adrenaline and nasal spray use. This approach might improve access for those with limited mobility or living far away from regional centres, as well as bringing convenience and cost savings for the patient and service provider. These potential benefits need to be carefully weighed against evidence of service safety and quality. Keys to success include delineation of appropriate clinical scenarios, patient selection, training, IT support and robust information governance framework. Welldesigned prospective studies are needed to evaluate its role.

Key words: telemedicine, allergy, allergy service, governance, patient education 


\section{Introduction}

The use of telecommunications in medicine is nothing new; the first telephone exchange in Glasgow in 1879 was specifically installed for doctors. However, recent rapid advances in the information technology (IT) industry have had a remarkable global impact on the lives of the public and it is inevitable that this will influence health service delivery options and expectations amongst patients and health care professionals. Importantly, IT has enabled health care professionals to store and transmit clinical data in order to expedite clinical decision-making within and between primary, secondary and tertiary care.

Telemedicine is the use of telecommunication and IT in the delivery of health and clinical care at a distance or remotely. This could be without the patient physically meeting the health care professional during the entire treatment pathway but is more commonly used in specific clinical scenarios or certain aspects of management following initial evaluation.

The main objective of this review is to consider whether current models of telemedicine could be applied to clinical allergy services. These models may help address the unmet need for these services, which is further compounded by recent re-structuring of specialist services and the associated financial squeeze within the United Kingdom (UK) National Health Service (NHS).

\section{Types of telemedicine}

Broadly speaking telemedicine can be offered using 2 modalities singly or in combination:

1. Store-and-Forward: This involves transmission of packets of data relating to investigations such as digital images in radiology/pathology, use of high resolution digital cameras in dermatology, digital otoscopes, digital stethoscopes and physiological parameters such as blood pressure (BP) measurements, 24 hour ECG and peak expiratory flow rate recordings. The specialist receiving these data can offer 
diagnostic input and management advice without the need for the patient to travel.

Provision of specific, relevant clinical information to the specialist are interpreted in the context of the clinical presentation.

2. Real-time interactive Services: This involves remote but real time contact using IT tools such as telephone or video calling (eg: Skype) to enable remote consultation. It follows that this modality depends on availability of relevant technology to the specialists and patients as well as possession of relevant IT skills by both parties. This has been applied in dermatology, psychology, psychiatry and nursing, as well as in the follow-up of chronic conditions such as diabetes, asthma and heart disease with an option for face-to-face (FTF) consultation if deemed necessary. This method is clearly not suitable for conditions that require a detailed physical examination.

Remote monitoring or telemetry may also be an useful tool_for patients with chronic conditions such as in diabetes mellitus, asthma and cardiac failure following an initial FTF clinical assessment by a specialist. Monitoring is done on the basis on specific physiological variables or point of care tests, symptomatic responses and quality of life scores. This modality relies on availability of equipment for home use (eg: BP monitor, home glucometer, electronic peak flow device) and patients ability to perform the relevant tests unsupervised at home and following instructions. Hence, successful implementation requires patient cooperation, acquisition of skills and suitable mental and physical well-being to cooperate and perform the relevant task/s and generate data (http://www.peninsulacommunityhealth.co.uk/our-services/telehealth.htm,accessed 04/08/2015). Increasingly patient monitoring devices are able to acquire and transmit data in real time wirelessly with reduced need for patient intervention. 


\section{Potential benefits of telemedicine}

From the patients perspective, telemedicine enables access to health services without having to travel to the specialist. It is therefore particularly relevant to patients in remote areas with poor access to health care or to those who are unable to travel due to transport or mobility problems, other disabilities, financial restrains or work commitments. From a health service provider view point, using telemedicine to deal with some of the cases referred to a specialist should reduce the cost of assessing and monitoring a wide range of conditions and reduce the number of cancelled and wasted appointments. Telemedicine can also be applied to health education for patients and health care professionals.

\section{Applications of Telemedicine}

Several studies have investigated the possible role of telemedicine in clinical practice. Whilst preliminary data are promising, the quality of evidence available thus far is limited as it mainly comes from observational studies with relatively small sample sizes. There is paucity of information relating to cost-effectiveness and a lack of sound research methodology with only a small number of adequately powered randomised control trials (RCTs).

Surveys carried out amongst general practitioners (GPs) have suggested willingness to consider alternatives to conventional FTF consultations if support for IT and guidance on medico-legal advice is accessible(1). One study reported that GPs use email to communicate with patients and that this occurs in an unregulated and unstructured manner which is not compatible with the high standards set out by clinical governance frameworks in the UK NHS(2) and elsewhere in Europe, USA, Australia and New Zealand.

In a survey of 713 patients in 5 primary care practices in Missouri, Columbia, USA, 78\% were internet users, although $36.7 \%$ of respondents could not afford to pay for an internet connection (3). Lack of IT skills and access to computers were the main barriers amongst 
non-users. Predictably, perhaps younger age, higher education, higher income, better health or absence of chronic illness were associated with internet use(3). This is an obvious barrier to reaching those patients who would benefit most from internet-based telemedicine services, and means that the role of older technology (the telephone [landline or mobile]) will remain important. A Cochrane systematic review showed that telephone consultations used in primary care significantly reduced the number of contact visits and 'out of hours' calls for GPs(4). In a North American study carried out in 35,423 patients with hypertension, diabetes or both, use of a secure communication between patients and their physicians for clinical management-related enquiries was associated with improved Health Care Effectiveness Data and Information Set (HCEDIS) measures(5). In a randomised trial carried out in the UK of joint tele-consultations (virtual outreach) versus standard outpatient appointments for patients referred by their GP for specialist opinion, more patients in the former group were offered follow up appointments. This was particularly so for the Ear Nose and Throat department and Orthopaedics as opposed to other specialties(6). Patient satisfaction was greater in the telemedicine group(6), however the cost incurred to the NHS for the telemedicine group was greater, although it saved time and money for the patients(7).

The financial aspects incurred in running a telemedicine service may have reduced significantly in the last 10 years with the rapid proliferation of the IT industry, resulting in a greater proportion of the global population using smart phones and portable computers. Furthermore, most hospitals in the UK NHS are moving towards digital technology and paperless systems and these would reduce the capital costs for the establishment of telemedicine services. In summary, preliminary evidence on the use of telemedicine in primary care seems promising giving an impetus for carrying out well designed large RCTs.

Teleradiology has also shown some encouraging results. Introduction of teleradiology in in the island of Ameland, off the coast of The Netherlands resulted in a reduced number of 
missed fractures and a reduction in unnecessary referrals(8). Similarly, teleradiology guided thrombolysis for acute stroke achieved comparable results to 'on-site' treatment decisions. A high degree of correlation was also seen with respect to decisions on coronary angiograms made by transmission of digital images to smart phones via Face Time application compared to conventional on site reporting in the laboratory $(9,10)$.

Legget et al reported high diagnostic accuracy (97\%) for rheumatological conditions using real time televisual consultation with $58 \%$ of patients expressing a preference for telemedicine consultation(11). Similar studies support use of telemedicine in long term management of diabetes(12-14).

Teledermatology has been particularly successful, perhaps because diagnosis is often arrived on the basis of visual examination of skin lesions with less emphasis on extended historytaking or detailed physical examination. Studies examining teledermatology have employed Store-and-Forward as well as real time interactive clinical services using audio-visual conferencing(15-20) and reported high diagnostic accuracy, good clinical outcomes, patient satisfaction and preference for telemedicine.

Employing telemedicine in intensive care units (ICU) in the USA has been driven by need rather than by choice. In 2010, there was a mismatch of 5,500 American board-certified intensivists for 6,000 ICUs in the USA(21). Tele-ICU has an off-site command centre in which a critical care team comprising an intensivist and critical care nurse are linked remotely to the patient with real time audio, video and transmission of clinical data to offer support to less skilled bed side staff regarding management decisions. Preliminary evidence suggests there is reduction in length of ICU stay and mortality, but further studies are required to confirm the value of such a service in critically ill patients(21, 22). There is also 
emerging evidence favouring use of tele-cardiology in long term clinical management of paediatric and adult patients with heart failure and arrhythmias (23-25).

In contrast, studies investigating the use of telemonitoring in chronic obstructive pulmonary disease (COPD) and asthma did not show favourable results. Telemonitoring failed to achieve any reduction in rates of COPD exacerbations(26). In asthma, telemedicine has mainly focussed on monitoring treatment plans and electronic monitoring devices (EMDs) to promote compliance. A systematic review and meta-analysis of 6 studies involving telephone, email or monitoring (from 813 articles) did not support the role for telemedicine interventions to improve asthma outcomes, although they could not exclude other benefits(27). The authors concluded that further studies are needed to assess the role for EMDs in clinical outcomes, cost effectiveness and patient selection in management of chronic asthma(28). This observation that telemedicine does not inevitably improve outcomes reinforces the need for systematic trials in each proposed clinical application.

\section{Potential barriers for use of telemedicine}

The use of telemedicine in clinical practice depends on multiple factors, some of which are specific to patients and health care professionals. Provision of appropriate resources including technical support and training for the relevant IT tools would be key for the service provider and the patient. Staff delivering telemedicine services must be trained to ensure compliance with Caldicott principles and the Data Protection Act of 1998. Importantly, development of an appropriate governance framework surrounding use of telemedicine is essential for medico-legal purposes, data protection and patient confidentiality. Specific purpose-built tools and governance systems will be necessary. There may also be barriers erected locally by organisations and staff who resist or oppose the introduction of telemedicine since it threatens to bring changes to working practices. There may also be 
issues with adequate internet connectivity in rural or isolated areas where patients benefit the most from telemedicine. These concerns highlight the need for well-designed RCTs and cost-effectiveness studies.

\section{Is there a role for telemedicine in adult allergy practice in the UK NHS ?}

The organisation of the UK NHS adult allergy services differs both from allergy services in other countries and also from other specialties in the UK. It is delivered by allergists, clinical immunologists, respiratory physicians, dermatologists, Ear Nose and Throat (ENT) surgeons, dermatologists, gastroenterologists and GPs with a special interest. Also, there are variations with respect to professional training, availability of resources and infrastructure of clinical services and geographical distribution of clinics. The unmet need for allergy services in the UK has been highlighted in several publications including those under the auspices of The Royal College of Physicians and House of Lords, Science and Technology

Committee reports(29, 30). Given the restructuring of specialist services within the UK NHS in recent years, campaigns for equity of health care for all, budget cuts and high expectations of patients, there is a real need to be innovative in creating new service frameworks for delivery of allergy services.

Allergy diagnosis depends largely on information derived from a comprehensive clinical history and scrutiny of records relating to previous admissions or other clinical events involving suspected allergic episodes (e.g. emergency department or anaesthetic records). The experience of the authors in a large UK regional allergy service is that in vivo diagnostic tests are required only in a small proportion of adults patients referred to the allergy clinic. In other words, a significant proportion of referrals relate to adult patients whose symptoms are not in keeping with an IgE-mediated condition or where clinical history supports a spontaneous (idiopathic) aetiology such as chronic urticaria. Further scenarios where allergy 
testing is often unnecessary include food intolerance, adverse reactions to some drugs, aspirin or non-steroidal anti-inflammatory drug (NSAID) intolerance or a spontaneous or druginduced anaphylaxis where the diagnosis can be arrived at through a careful clinical history. This highlights a potential role for telemedicine in triaging the patient following receipt of a referral and enabling selection of patients who require further investigations including skin tests and challenges in the clinic.

There have been significant advances in in vitro diagnostics including molecular allergology in the last decade and a case could be made for applying telemedicine alongside in vitro diagnostic tests in specific clinical scenarios (eg: allergic rhinitis, hay fever, nut allergy, oral allergy syndrome, seafood allergy). This approach could enable remote diagnosis and implementation of management plans. A combination of a purpose built package of Storeand-Forward and real time interactive services could be applied in such cases using tools such as email, telephone consultation and video calls or conferencing. Patient education (eg: adrenaline auto-injector training) can also be delivered using online materials or applications downloaded to smart phones or other portable devices.

In the following sections we propose some models of care using telemedicine, which can be applied to specific clinical scenarios in UK adult allergy practice. These may be a means to optimise efficient use of the limited resources and meet patient satisfaction without compromising quality of care. Investigations and management would also remain consistent with British Society for Allergy and Clinical Immunology (BSACI) (31, 32) and National Institute of Health Care and Excellence (NICE) guidelines (http://guidance.nice.org.uk/CG134). This approach is based on a triaging system, so that only selected cases (which we estimate would be a relatively small proportion) will require a further FTF consultation in clinic. This would reduce overhead expenses, travel time and expenditure for patients and for the most part management plans could be implemented in 
liaison with primary care. Patients could be counselled remotely and provided with educational materials, either in hard copy or via a validated web tool and make it a paperless exercise. This approach would require prior agreement of referral criteria and pathways with relevant stake holders.

Essential pre-requisites of a telemedicine service are summarised in Table-1. Importantly, the bench mark for IT systems should be predefined and standardised amongst the telemedicine vendors to maintain equity. Furthermore, the clinical scenarios in which telemedicine will be applied should be agreed with local authorities. Standard operating protocols should be developed and these should be subjected to regular audits and patient satisfaction surveys. This service should be piloted initially and clinic schedules should be based on availability of space, IT support and personnel availability.

The following proposed models of care need validation in well-designed prospective studies:

1. Model 1: Triaging system for allergic rhinitis and hay fever referrals: A structured questionnaire is made available online or in hard copy, which enables GPs to select patients for referral to an allergy specialist for allergen-specific immunotherapy. Alternatively, a combined approach of telephone consultation and a Store-andForward systems may be employed by the specialist to triage patients for allergenspecific immunotherapy (Figure-1).

2. Model 2: Triaging for suspected cases of anaphylaxis discharged from accident and emergency departments and remote training (Figure-2):

We (33) and others $(34,35)$ have shown that a significant proportion (50-70\%) of adult anaphylaxis presenting to emergency units is triggered by drugs (antibiotics, NSAIDs) or is spontaneous (idiopathic) in aetiology and neither scenario requires allergy testing. Management plans usually involve counselling of patients, checking baseline serum 
tryptase, discussing avoidance measures (where relevant) and provision of training in the use of self-injectable adrenaline pens. A recent survey of 100 NHS Allergy clinics showed that only $52 \%$ offered annual re-training in the use of self-injectable adrenaline pens, with some citing lack adequate resources and a high demand for the service(36). In a significant proportion of patients competence and skill in the use of adrenaline pen is lacking, underlining the need for periodic re-training(37-40). Furthermore, the need to train healthcare professionals involved in the management of anaphylaxis has also been highlighted previously(40). Telemedicine tools may offer a solution through remote training for patients and healthcare professionals. Real time interactive telemedicine services such as video-conferencing could be employed and educational materials made available through online videos, or applications can be downloaded onto smart phones or other portable devices. Certain food allergies could also be managed using a similar approach using molecular allergy tests via primary care to confirm the diagnosis (eg: peanut allergy or wheat-dependent exercise-induced anaphylaxis). Patients requiring skin tests for specific foods, drugs or challenge testing, those warranting venom immunotherapy and those with complex clinical presentations such as somatisation disorder or vocal cord dysfunction (VCD) could be invited for a FTF consultation.

3. Model 3: Triaging for suspected food allergy: A triaging system is also likely to work in this clinical scenario since the need for skin tests or in vitro diagnostics or both is based on clinical presentation. Hence, a clinical history could be obtained by a real-time interactive approach and cases triaged for further investigations including skin tests and challenges. Patients would be counselled regarding non-allergic conditions such as food intolerance, and those with suspected type-1 hypersensitivity could undergo in vitro diagnostics via primary care. If food allergy is confirmed, the 
patient is counselled further regarding avoidance measures and provision of training regarding adrenaline auto-injector as discussed under model 2.

4. Model 4: Remote management of chronic urticaria (Fiqure-3): Telemedicine has been shown to be successful and real-time interactive services could be applied in patients with chronic urticaria with a view to confirming diagnosis and providing educational materials. Advice and guidelines to the GP for optimisation of therapy with high dose antihistamines and leukotriene antagonists could be delivered in paper format or via online links. Patients who require immunomodulatory therapies such as omalizumab or cyclosporine would be invited for a FTF consultation.

One of the challenges with this telemedicine approach is to develop a robust and validated method of obtaining a structured clinical history by either an interview using telephone or video call, or an electronic questionnaire to be completed either by the patient or their GP. These have to be condition-specific and provide the necessary data set for triaging and making clinical decisions as discussed above.

The development of a standard set of questions firstly involves review of questions currently used to obtain clinical histories across clinics and clinicians for different conditions or a given clinical scenario. A comprehensive and inclusive question set could then be drawn up and used in consultations alongside diagnostic tests (where relevant). Reliability could be demonstrated by different clinicians arriving at the same diagnosis for a patient based on the analysis of answers to the clinical history question set (and diagnostic tests where relevant). Reliability over time could also be demonstrated if the diagnosis for a patient is the same at two different time points (provided there is no clinical change in the patient). This standardised question set should then be used in telemedicine consultations and in FTF consultations to check that diagnosis made using the two different modes are correlated. 
This approach may not work for all patients since an open consultation with an opportunity to clarify and discuss symptoms may be warranted in some with relatively complex symptomatology or in those with psychosomatic or somatisation disorder.

There are a number of measures that would be useful to use in an assessment of the feasibility, acceptability and usefulness of a telemedicine allergy service. Some measures, such as quality of life, might already be available and would need to be assessed for the validity of their use in a telemedicine context. Others, such as patient satisfaction with an allergy telemedicine service, would need to be developed. Development of this type of patient relevant outcome measure (PROM) should also follow standard guidelines (where available), which would involve interviews with patients to discuss their views on such a service, analysis of interview data to develop a set of patient satisfaction questions which would then be given to a large number of patients using the service. Similarly, the views of clinicians on their experiences and confidence when using telemedicine for allergy should also be collected and evaluated.

Any study which aims to assess the feasibility of telemedicine for allergy would also need to develop a governance framework for delivery of the proposed models of care. An important consideration is the security of any data or photographs that might be sent via electronic means. The means of transport and storage of the data should be secure, the duration of data storage and access permissions would need to be decided. Confidentiality of patient data is paramount in any system set up to provide telemedicine consultations. The confidentiality of the consultation itself should be addressed, and patients may not have considered the issues involved in advance. The patient may talk to their consultant via a telephone or computer from their home while others are present, such as children, other family members or friends. Although it should be the patients choice as to who is present during a consultation, they should be made aware that issues might come up during a consultation that they do not want 
others to hear. It is also wise for the clinician to be aware of who else is present, since this may influence the answers given to questions. These factors are naturally apparent in FTF consultations, but require extra care and consideration in telemedicine. When considering a governance framework for telemedicine (Table-2) for allergy, ways in which the safety of the system in terms of security and confidentiality can be clearly communicated to the patient should be developed in order to provide the patient with a level of confidence in such a service. 


\section{Conclusion}

Telemedicine presents an attractive option for the adult allergy service. Well-designed prospective studies are needed to evaluate its role in specific clinical conditions. These should take into account health economics, impact on health-related quality of life and patient and clinician satisfaction, to aid policy makers in making informed decisions on whether to adopt this approach. Keys to the success of this approach include delineation of appropriate clinical contexts, patient selection, training, IT support and robust information governance framework. 


\section{Disclosures}

MTKs and APHs department have received educational grants from ALK Abello, Thermo Fisher and MEDA pharmaceuticals in the recent years for PracticAllergy course. MTK has following roles: (a) chair/clinical lead of Improving Quality in Allergy Services (IQAS), Royal College of Physicians national accreditation programme for adult allergy services (b) audit lead for BSACI (c) member of Standards of Care Committee and Adult Allergy Group, BSACI (d) member of national Clinical Reference Group for specialised services in Allergy and Immunology. 


\section{REFERENCES}

1. Hanna L, May C, Fairhurst K. The place of information and communication technologymediated consultations in primary care: GPs' perspectives. Fam Pract 2012;29:361-366

2. Atherton H, Pappas $\mathrm{Y}$, Heneghan $\mathrm{C}$, Murray E. Experiences of using email for general practice consultations: a qualitative study. Br J Gen Pract 2013;63:e760-767

3. Kruse RL, Koopman RJ, Wakefield BJ, Wakefield DS, Keplinger LE, Canfield SM, Mehr DR. Internet use by primary care patients: where is the digital divide? Fam Med 2012; 44:342347

4. Bunn F, Byrne G, Kendall S. Telephone consultation and triage: effects on health care use and patient satisfaction. Cochrane Database Syst Rev 2004(4):CD004180

5. Zhou YY, Kanter MH, Wang JJ, Garrido T. Improved quality at Kaiser Permanente through email between physicians and patients. Health Aff (Millwood) 2010; 29:1370-1375

6. Wallace P, Haines A, Harrison R, Barber J, Thompson S, Jacklin P, Roberts J, Lewis L, Wainwright. Joint teleconsultations (virtual outreach) versus standard outpatient appointments for patients referred by their general practitioner for a specialist opinion: a randomised trial. Lancet 2002;359:1961-1968

7. Jacklin PB, Roberts JA, Wallace P, Haines A, Harrison R, Barber JA, Thompson SG, Lewis L, Currell R, Parker $S$, Wainwright $P$. Virtual outreach: economic evaluation of joint teleconsultations for patients referred by their general practitioner for a specialist opinion. BMJ 2003;327:84

8. Jacobs JJ, Jacobs JP, van Sonderen E, van der Molen T, Sanderman R. Fracture diagnostics, unnecessary travel and treatment: a comparative study before and after the introduction of teleradiology in a remote general practice. BMC Fam Pract 2015; 16:53

9. Fong WC, Ismail M, Lo JW, Li JT, Wong AH, Ng YW, Chan PY, Chan AL, Chan GH, Fong KW, Cheung NY, Wong GC, Ho HF, Chan ST, Kwok VW, Yuen BM, Chan JH, Li PC. Telephone and Teleradiology-Guided Thrombolysis Can Achieve Similar Outcome as Thrombolysis by Neurologist On-site. J Stroke Cerebrovasc Dis 2015; 24:1223-1228

10. Bilgi M, Erol T, Gullu H, Sezgin AT, Hamad S, Bilgel ZG, Muderrisoglu H. Teleconsultation of coronary angiograms using smartphones and an audio/video conferencing application. Technol Health Care 2013; 21:407-414

11. Leggett P, Graham L, Steele K, Gilliland A, Stevenson M, O'Reilly D, Wootton R, Taggart A. Telerheumatology--diagnostic accuracy and acceptability to patient, specialist, and general practitioner. Br J Gen Pract 2001;51:746-748

12. Izquierdo RE, Wang D, Huang D, Palmas W, Weinstock RS. Case Management with a Diabetes Team Using Home Telemedicine: Acceptance of Treatment Recommendations by Primary Care Providers in IDEATel. Telemed J E Health 2015 (Jun26th).

13. Carallo C, Scavelli FB, Cipolla M, Merante V, Medaglia V, Irace C, Gnasso A. Management of Type 2 Diabetes Mellitus through Telemedicine. PLoS One 2015 10:e0126858

14. Rasmussen BS, Froekjaer J, Bjerregaard MR, Lauritsen J, Hangaard J, Henriksen CW, Halekoh U, Yderstraede KB. A Randomized Controlled Trial Comparing Telemedical and Standard Outpatient Monitoring of Diabetic Foot Ulcers. Diabetes Care 2015;June 26th

15. Nami N, Massone C, Rubegni P, Cevenini G, Fimiani M, Hofmann-Wellenhof R. Concordance and Time Estimation of Store-and-forward Mobile Teledermatology Compared to Classical Face-to-face Consultation. Acta Derm Venereol 2015; 95:35-39

16. Lamel S, Chambers CJ, Ratnarathorn M, Armstrong AW. Impact of live interactive teledermatology on diagnosis, disease management, and clinical outcomes. Arch Dermatol 2012;148:61-65

17. Lamel SA, Haldeman KM, Ely H, Kovarik CL, Pak H, Armstrong AW. Application of mobile teledermatology for skin cancer screening. J Am Acad Dermatol 2012;67:576-581 
18. Al Quran HA, Khader YS, Ellauzi ZM, Shdaifat A. Effect of real-time teledermatology on diagnosis, treatment and clinical improvement. J Telemed Telecare 2015;21(2):93-9.

19. Fogel AL, Teng JM. Pediatric teledermatology: a survey of usage, perspectives, and practice. Pediatr Dermatol 2015;32:363-368

20. Warshaw EM, Gravely AA, Nelson DB. Reliability of store and forward teledermatology for skin neoplasms. J Am Acad Dermatol 2015;72:426-435

21. Kumar S, Merchant S, Reynolds R. Tele-ICU: Efficacy and Cost-Effectiveness Approach of Remotely Managing the Critical Care. Open Med Inform J 2013;7:24-29

22. van der Voort PH, de Metz J, Wester JP, van Stijn I, Feijen HM, Balzereit A, Rijkenberg S, Obster R, Bosman RJ. Telemedicine in a Dutch intensive care unit: A descriptive study of the first results. J Telemed Telecare $2015 \mathrm{Jul} 12$

23. Lopez-Magallon AJ, Otero AV, Welchering N, Bermon A, Castillo V, Duran A, Castro J, Munoz R . Patient Outcomes of an International Telepediatric Cardiac Critical Care Program. Telemed J E Health 2015;21:601-610

24. Piotrowicz E, Stepnowska M, Leszczynska-Iwanicka K, Piotrowska D, Kowalska M, Tylka J, Piotrowski W, Piotrowicz R. Quality of life in heart failure patients undergoing home-based telerehabilitation versus outpatient rehabilitation--a randomized controlled study. Eur J Cardiovasc Nurs 2015;14:256-263

25. McCrossan BA, Doherty NN, Sands AJ, Grant B, Craig BG, McCusker CG, Casey FA. Survey of paediatricians' opinions on a regional paediatric telecardiology service. J Paediatr Child Health 2014;50:482-486

26. Pinnock H, Hanley J, McCloughan L, Todd A, Krishan A, Lewis S, Stoddart A, van der Pol M, MacNee W, Sheikh A, Pagliari C, McKinstry B. Effectiveness of telemonitoring integrated into existing clinical services on hospital admission for exacerbation of chronic obstructive pulmonary disease: researcher blind, multicentre, randomised controlled trial. BMJ 2013;347:f6070

27. Zhao J, Zhai YK, Zhu WJ, Sun DX. Effectiveness of Telemedicine for Controlling Asthma Symptoms: A Systematic Review and Meta-analysis. Telemed J E Health 2015;21:484-492

28. Chan AH, Reddel HK, Apter A, Eakin M, Riekert K, Foster JM. Adherence monitoring and ehealth: how clinicians and researchers can use technology to promote inhaler adherence for asthma. J Allergy Clin Immunol Pract 2013;1(5):446-454

29. Royal College of Physicians. 2003 Allergy: the unmet need.

30. House of Lords. Science and Technology Committee. 6th Report of session 2006-7 Allergy Volume 1: Report.

31. Powell RJ, Leech SC, Till S, Huber PA, Nasser SM, Clark AT. BSACl guideline for the management of chronic urticaria and angioedema. Clin Exp Allergy 2015;45:547-565

32. Krishna MT, Ewan PW, Diwakar L, Durham SR, Frew AJ, Leech SC, Nasser SM. Diagnosis and management of hymenoptera venom allergy: British Society for Allergy and Clinical Immunology (BSACl) guidelines. Clin Exp Allergy 2011;41:1201-1220

33. Buka R CR, Melchior C, Huissoon AP, Hackett S, Dorrian S, Cooke MW, Krishna MT. Anaphylaxis and Ethnicity: Higher Incidence in British South Asians. Allergy 2015;Jul 27 th (epub ahead of print)

34. Brown AF, McKinnon D, Chu K. Emergency department anaphylaxis: A review of 142 patients in a single year. J Allergy Clin Immunol 2001;08:861-866

35. Moro Moro M, Tejedor Alonso MA, Esteban Hernandez J, Mugica Garcia MV, Rosado Ingelmo A, Vila Albelda C. Incidence of anaphylaxis and subtypes of anaphylaxis in a general hospital emergency department. J Investig Allergol Clin Immunol 2011; 21:142-149

36. Krishna MT, Huber PA, Nasser SM, Ewan PW, Clark AT. Prescription of Adrenaline AutoInjectors - A National Survey of UK Allergy Specialists. Clin Exp Allergy 2015; Sept19th (epub ahead of print). 
37. Diwakar L, Heslegrave J, Richter AG, Huissoon AP, Krishna MT. Self-injectable adrenaline devices: is training necessary? J Investig Allergol Clin Immunol 2010;20:452-453

38. Sicherer SH, Forman JA, Noone SA . Use assessment of self-administered epinephrine among food-allergic children and pediatricians. Pediatrics 2000;105:359-362

39. Skorpinski EW, McGeady SJ, Yousef E. Two cases of accidental epinephrine injection into a finger. J Allergy Clin Immunol 2006;117:463-464

40. Grouhi M, Alshehri M, Hummel D, Roifman CM. Anaphylaxis and epinephrine auto-injector training: who will teach the teachers? J Allergy Clin Immunol 1999;104:190-193 


\section{Table-1: Pre-requisites of a telemedicine service and research}

\section{$\underline{\text { Patient related factors }}$}

1. Suitable patient - capable and willingness to accepting of phone or online consultation and possession of IT skills

2. Access to telephone, personal computer with high-speed internet access

\section{$\underline{\text { Service Provider }}$}

1. Staff training and protected time

2. Quiet room with telephone, personal computer with facilities for video calls and high speed internet

3. Clear governance procedures in place

4. Secure network to protect patient confidentiality

5. IT support

6. For Store-and-Forward service: good IT facilities for transmission of large files (e.g. high resolution images) in an encrypted format

\section{Qualitative Assurance}

1. Validated structured interview or questionnaire

2. Validated patient educational tools

3. 'Fit for purpose' audit tools

4. Pre-agreed referral pathways with contingency plans for FTF consultations where needed

5. Tools for measuring clinical outcome

6. Tools for measuring cost-effectiveness

7. Tools for measuring patient satisfaction 


\section{Table-2: Governance issues relating to remote communication with patients}

1. Informed consent should be sought from the patient prior to using any remote messaging or consultation according to local policies.

2. Minimum required patient identifiable material should be used during communication

3. Patients should be made aware of:

o potential confidentiality and security breaches during information transit

o potential messaging errors and modifications due to IT-related issues

o potential interception of information by friends, family members or members of public who may have access to patients email account or mobile phone

- Service disruptions of mobile phone, internet or email servers may potentially affect clinical management

\section{Issues relating to SMS messaging and email patient communication}

4. Patients should be advised to delete the messages from phones or email servers when they are no longer required

5. All such communication should be included in the patients clinical record

\section{Issues relating to telephone and video-call consultations}

6. The patient should have an option to have a FTF consultation in addition to or instead of the remote consultation

7. The patient should be advised of their right to privacy and how to maintain this in a remote consultation

8. All verbal discussions and instructions should be recorded in written form (as in FTF consultation) 


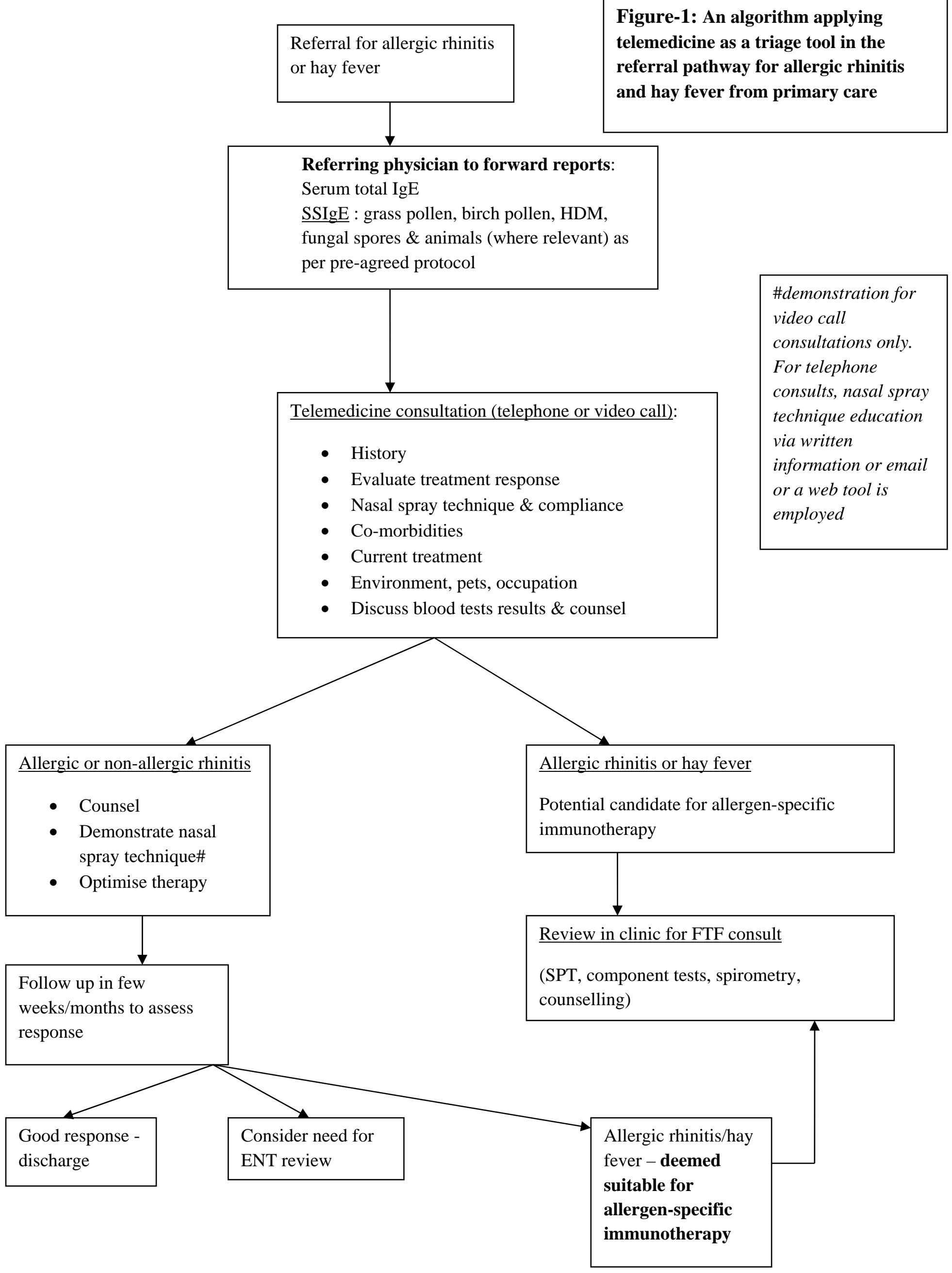


